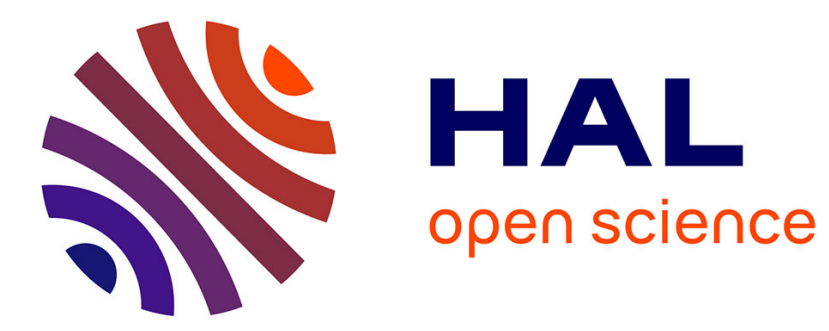

\title{
Event-triggered observer design for delayed output-sampled systems
}

Chengcheng Song, Haoping Wang, Yang Tian, Gang Zheng

\section{To cite this version:}

Chengcheng Song, Haoping Wang, Yang Tian, Gang Zheng. Event-triggered observer design for delayed output-sampled systems. IEEE Transactions on Automatic Control, 2020, 65 (11), pp.4824 4831. 10.1109/TAC.2019.2960267 . hal-02406816

\section{HAL Id: hal-02406816 https://hal.inria.fr/hal-02406816}

Submitted on 12 Dec 2019

HAL is a multi-disciplinary open access archive for the deposit and dissemination of scientific research documents, whether they are published or not. The documents may come from teaching and research institutions in France or abroad, or from public or private research centers.
L'archive ouverte pluridisciplinaire HAL, est destinée au dépôt et à la diffusion de documents scientifiques de niveau recherche, publiés ou non, émanant des établissements d'enseignement et de recherche français ou étrangers, des laboratoires publics ou privés. 


\title{
Event-triggered observer design for delayed output-sampled systems
}

\author{
Chengcheng Song, Haoping Wang, Senior Member, IEEE, Yang Tian, and Gang Zheng
}

\begin{abstract}
This paper investigates the event-triggered observer design problem for nonlinear state affine systems. The output data of systems are event-triggered-sampled (sparse) and received by observer with certain time delays. A novel event-triggered mechanism is proposed to decide when the data need to be transmitted. The proposed observer globally and exponentially converges to the considered plant under the designed event-triggered mechanism. The effectiveness of the proposed event-triggered observer is illustrated with the comparison to existing works through simulations.
\end{abstract}

Index Terms-Event-triggered, observer, nonlinear, time delays.

\section{INTRODUCTION}

In conventional networked control systems, data are sampled and transmitted periodically for the communication of different components. Recently, the aperiodic event-triggered sampling and transmission method becomes a rising concern due to the capacity of saving energy, such as bandwidth and sensor battery energy. The key of such a method is to design an event-triggered mechanism with which the stability of the investigated system can be still guaranteed.

The problems of event-triggered control systems with full state available have been abundantly studied in the literature [1]-[5], including observer-based control techniques. However, most of these studies are limited only to linear systems [6]-[13], and few papers deal with nonlinear systems [14]-[22]. Precisely, [14], [15] studied an event-triggered mechanism in which the data are sent when the error induced by the event-triggered sampling exceeds a prescribed threshold, and this threshold was replaced by a time-varying one in [16]-[22]. In those mentioned papers, the common idea was to design the event-triggered mechanism in a common fixed form, depending on the dynamics of the data to be transmitted. Actually, most of studies in the event-triggered transmission adopt this conception to design the event-triggered mechanism. For example, [23][25] studied the event-triggered sampling problem with time delays based on this conception. Even though this conception provides the convenience and simplicity for controller design, it still provides some conservative conditions: it imposes more transmissions when the system's trajectory changes fast, and less transmissions when it changes slowly. A more reasonable even-triggered mechanism might be dependent of the system's performance. Thus, a new eventtriggered mechanism depending on system's performance is proposed in this paper, based on which an impulsive observer is designed for a class of nonlinear systems. The key conception is to design the event-triggered mechanism, based on the system's performance requirement, to yield less restrictive condition. In fact, this conception has already attracted researchers' attention, for example [12], [26], [27], however those studies were incomplete and unsystematic. [12] studied only linear systems, while the method in this paper is for a class of nonlinear systems. The result stated in [26] did not consider

Chengcheng Song, Haoping Wang, and Yang Tian are with the SinoFrench International Joint Laboratory of Automatic Control and Signa Processing, School of Automation, Nanjing University of Science \& Technology, Nanjing 210094, China (e-mail:Schengcheng@126.com, Corresponding author: hp.wang@njust.edu.cn, tianyang@njust.edu.cn)

Gang Zheng is with Inria Lille, France (e-mail:gang.zheng@inria.fr) the time delay which will be studied in this paper. Although the event-triggered mechanism has also been studied in [27] for nonlinear systems, the system performance based conception was only used to design a parameter of the event-triggered mechanism, instead of designing the whole event-triggered mechanism.

The observer design of sampled-data system mainly has three methods, including continuous-time method [28]-[30], discrete-time method [31], and hybrid method [32], [33]. Generally, the observer designed via the continuous-time method finally needs to be digitized in practical applications, Consequently, the sampling period cannot be too large. The observer designed via the discrete-time method needs an exact discrete-time model of systems, which is usually not suitable for nonlinear systems. Thus, the hybrid method is more appropriate for dealing with the observer design problem for sampleddata nonlinear systems, compared with the other two methods. The idea of this paper is based on the hybrid observer proposed in [33], which in fact was an extension of the result published in [34]. In our work, an event-triggered observer for nonlinear state affine systems in the networked environment is proposed. Due to the limited communication resources, the system's output is eventtriggered-sampled (sparse) and received by the observer from an unknown network, inducing unknown time delays. Based on the received data, an observer will be designed according to system performance requirement with a novel event-triggered mechanism.

In this paper, the following notations are used. The symbols $\mathbb{R}$ and $\mathbb{R}^{+}$denote the sets of real numbers and positive real numbers, respectively. The notation $\|x\|$ represents the Euclidean norm of an element $x \in \mathbb{R}^{n}, n \in \mathbb{N}$. For $p, q \in \mathbb{N}, \mathbb{R}^{p \times q}$ stands for the set of real matrices of dimension $p \times q$. For $n \in \mathbb{N}, I_{n} \in \mathbb{R}^{n \times n}$ is the identity matrix of dimension $n \times n$. For $p, q, m, n \in \mathbb{N}$, if $F \subset \mathbb{R}^{p \times q}$ and $G \subset \mathbb{R}^{m \times n}, \mathbb{C}^{0}(F, G)$ denotes the space of all continuous functions mapping from $F$ to $G$. For $Q \in \mathbb{R}^{m \times n}, m, n \in \mathbb{N}$, the notation $\|Q\|$ represents the $L_{2}$-norm of $Q . F^{\prime}$ represents the transpose of matrix/vector $F$. We say that $\alpha I_{n} \leq S \leq \beta I_{n}$ where $S \in \mathbb{R}^{n \times n}, n \in \mathbb{N}$ if $\lambda_{\min }(S) \geq \alpha$ and $\bar{\lambda}_{\max }(S) \leq \beta$ where $\lambda_{\min }(S)$ and $\lambda_{\max }(S)$ denote the smallest and the biggest eigenvalues of matrix $S$, respectively. For $\zeta: \mathbb{R} \rightarrow \mathbb{R}^{p \times q}$ and $t \in \mathbb{R}$, the notation $\zeta^{-}(t)$ denotes the left limit of $\zeta$ at instant $t$ if it exists. $t_{0}$ represents the initial time in the sequel. $t_{k}$ for $k \in \mathbb{N}$ represents the detection instant of the proposed event-triggered condition, satisfying $t_{k}-t_{k-1}=t_{e}$ where $t_{e} \in \mathbb{R}$ is a positive constant. $\bar{t}_{j}$ for $j \in \mathbb{N}$ is the sending instant (or the event-triggered instant). $\tau$ represents a known upper bound of the communication delay that may occur.

\section{Problem Formulation}

Consider the following class of systems:

$$
\left\{\begin{array}{l}
\dot{x}(t)=A(u) x(t)+b(u) \\
y(t)=C x(t)
\end{array}\right.
$$

where $x \in \mathbb{R}^{n}$ is the state vector, $u \in D \subset \mathbb{R}^{m}$ is the input vector, $D$ is a compact set, and $y \in \mathbb{R}^{p}$ is the output vector. The matrices $A \in \mathbb{C}^{0}\left(\mathbb{R}^{m}, \mathbb{R}^{n \times n}\right), b \in \mathbb{C}^{0}\left(\mathbb{R}^{m}, \mathbb{R}^{n}\right), C \in \mathbb{R}^{p \times n}$ are known, with $n, m, p \in \mathbb{N}$ and $x_{0}=x\left(t_{0}\right)$. The transition matrix of system (1) denoted as $\psi_{u} \in \mathbb{C}^{0}\left(\mathbb{R}^{m}, \mathbb{R}^{n \times l}\right)$ is known, with $l \in \mathbb{N}$. 
Definition 1: Consider the following system, for $t \in\left[t_{0}, \infty\right)$ :

$$
\left\{\begin{aligned}
\dot{x}(t) & =A(u) x(t) \\
y(t) & =C x(t)
\end{aligned}\right.
$$

with the transition matrix $\psi_{u}\left(\cdot, t_{0}\right)$, which satisfies $\psi_{u}\left(t_{0}, t_{0}\right)=I_{n}$. The bounded input $u$ is said to be regularly persistent, if there exist $t_{a}, t_{b} \in\left[t_{0}, \infty\right), \delta \in \mathbb{R}^{+}$, such that, for all $t \geq t_{b}$

$$
\int_{t}^{t+t_{a}} \psi_{u}^{\prime}\left(s, t_{0}\right) C^{\prime} C \psi_{u}\left(s, t_{0}\right) d s \geq \delta I_{n}
$$

Regularly persistent inputs ensure the studied system to be observable, see [35]. Thus, We need the following hypothesis.

Hypothesis 1: The input $u$ is regularly persistent and the constants $t_{a}$ and $\delta$ are known.

In this paper, it is assumed that the output of system (1) is eventtriggered sampled and delayed. Under the assumption that system (1) is observable, the objective of this paper is to propose an eventtriggered mechanism (ETM) and design an event-triggered observer, such that the proposed observer can globally and exponentially estimate the states of system (1) under the designed ETM.

As it has been explained, we are interested in how to design an efficient observer for (1) when its output is sampled and contains unknown delay. The word 'efficient' means that we want to use as less information of the output as possible. Normally, a sampling process is introduced before sending the measurement to observer through the network with unknown but bounded delay. This sampling process could be either periodic or aperiodic. In [33], the upper and lower bounds of the sampling period are obtained. For the periodic sampling case, since the upper bound of the sampling period is fixed, even for the case where the observer can perfectly converge at the required rate without triggers at some time instants, the measurement will still be sampled with the upper bound period and sent to the network. From the efficiency point of view, such a configuration is in fact not a good option. Logically, if the observer can converge at the required rate even without transmissions at some time instants, then these transmissions are unnecessary. In other words, a mechanism to judge the performance of observer is useful to increase the efficiency. Inspired by this idea, the periodic sampling process is modified in this paper by inserting an ETM, which is depicted in Fig. 1.

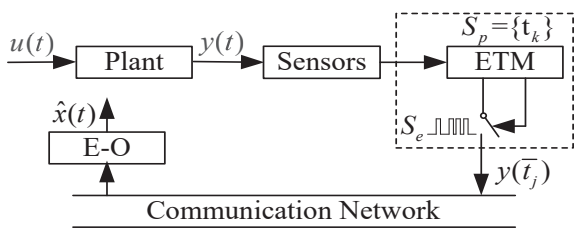

Fig. 1. Configuration of event-triggered observer (E-O) design for delay output-sampled system

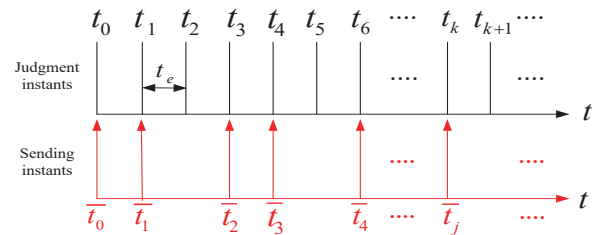

Fig. 2. Relationship between $t_{k} \in S_{p}$ and $\bar{t}_{j} \in S_{e}$

The proposed event-triggered mechanism is designed to decide whether to send the output data at each sampled instant $t_{k}$. That means the designed event-triggered mechanism actually belongs to the kind of periodic event-trigger mechanism, i.e. we consider periodic event trigger with delay. The notation $t_{k}$ for $k \in \mathbb{N}$ represents a strictly increasing sequence such that $\lim _{k \rightarrow \infty} t_{k}=\infty$ with $t_{k}-t_{k-1}=t_{e}, t_{e}>0$, which means it is periodic. Define $S_{p}=\left\{t_{k}\right\}$, which are the judgment instants for the event-triggered condition which will be exploited in Section III. In order to avoid the ambiguity, denote $S_{e}=\left\{\bar{t}_{j}\right\}$ as the set of sending instants, or eventtriggered instants, which are aperiodic. Obviously, one has $S_{e} \subseteq S_{p}$. The relationship between $t_{k} \in S_{p}$ and $\bar{t}_{j} \in S_{e}$ is shown in Fig. 2. Define $\tau$ as the known upper bound of the unknown transmission delay due to the communication network, and it is assumed in this paper that $\tau \leq t_{e}$. This assumption means that the sending data are available for the observer before the next sending instant. Therefore, the objective of this paper is to design an event-triggered observer, in which an event-triggered mechanism is designed to decide $\bar{t}_{j}$ when the stability of observer system is guaranteed, and these will be discussed in the next section.

\section{EVENT-TRIGGERED OBSERVER DESIGN}

\section{A. Impulsive observer}

For system (1), since $\tau$ is the upper bound of the unknown transmission delay due to the communication network, then the following event-triggered observer is proposed:

- for $k \geq 1, t \in\left[t_{k-1}+\tau, t_{k}+\tau\right)$

$$
\left\{\begin{array}{l}
\dot{\hat{x}}(t)=A(u) \hat{x}(t)+b(u) \\
\dot{S}(t)=-A^{\prime}(u) S(t)-S(t) A(u)-\mu S(t)
\end{array}\right.
$$

- for $j \geq 1, t \in\left[\bar{t}_{j-1}+\tau, \bar{t}_{j}+\tau\right)$

$$
\dot{w}(t)=C A(u(t-\tau)) \hat{x}(t-\tau)+C b(u(t-\tau))
$$

- for $t=t_{k}+\tau$

$$
\left\{\begin{array}{l}
S\left(t_{k}+\tau\right)=S^{-}\left(t_{k}+\tau\right)+t_{e} C^{\prime} C \\
\hat{x}\left(t_{k}+\tau\right)=\hat{x}^{-}\left(t_{k}+\tau\right) \\
+\rho t_{e} S^{-1}\left(t_{k}+\tau\right) C^{\prime}\left(w\left(t_{k}+\tau\right)-C \hat{x}\left(t_{k}\right)\right)
\end{array}\right.
$$

- for $t=\bar{t}_{j}+\tau$

$$
w\left(\bar{t}_{j}+\tau\right)=y\left(\bar{t}_{j}\right)
$$

where the notation $\hat{x} \in \mathbb{R}^{n}$ is the estimation of $x, S(t) \in \mathbb{R}^{n \times n}$ is the observation gain, $w(t) \in \mathbb{R}^{p}$ is the output estimation, $\mu \in \mathbb{R}^{+}$ and $\rho \in[1, \infty)$ are parameters which need to be designed. The Proposition 1 in the next part ensures the positive definiteness of matrix $S$, which guarantees the existence of $S^{-1} . \hat{x}_{0}$ and $S_{0}$ denote the initial conditions, and $S_{0}$ should be symmetric positive definite. Define $\hat{y}=C \hat{x}$ as the output of observer.

Note that $t_{k}$ is the sampling instant which is periodic and the period is determined based on Proposition 1 borrowed from [34]. $\bar{t}_{j}$ is the sending instant, which is aperiodic or event-triggered (See Fig. $2)$. Then it is clear that the proposed observer is composed of the continuous mode (4-5) and the discrete mode (6-7). At each discrete periodic instant $t_{k}+\tau$, an impulse is generated, and the observer is switched from continuous mode (4) to discrete mode (6), so the estimate of state is corrected with the output estimation $w\left(t_{k}+\tau\right)$. At each discrete aperiodic instant $\bar{t}_{j}+\tau$, an impulse is also generated, and the output estimator is switched from continuous mode (5) to discrete mode (7), so the estimate of output is corrected with the real output $y\left(\bar{t}_{j}\right)$.

Note that the above designed observer (4-7) contains only the known variables $t_{k}, \bar{t}_{j}$ and $\tau$, so a copy of the observer model can be set at the level of the event-triggered mechanism (or the sensor side). In other words, the states of the proposed observer $\hat{x}$ can be 
seen as the available information when designing the event-triggered mechanism. Actually, the similar idea has also been used to solve the problem of event-triggered control in the literature [36]. Since $\tau$ is known, which is the upper bound of unknown delays, a buffer is necessary to be set at the observer side, to store the received output data, and then update observer at time instant $\bar{t}_{j}+\tau$. To simplify the notations, the following regards $\hat{x}$ as the state of the observer designed at the level of ETM.

Since the variables in the proposed observer can be seen as the available information when designing the event-triggered mechanism, now we can start to design a new event-triggered mechanism. Define $V_{y}(t)=e_{y}^{\prime}(t) e_{y}(t)$ with $e_{y}=\hat{y}-y$. Since the judgment instants of the event-triggered condition are $t_{k} \in S_{p}$, the sending instants (or the event-triggered instants) $\bar{t}_{j} \in S_{e}$ are determined as follows:

$$
\begin{aligned}
S_{e}= & \left\{t \in S_{p} \mid\right. \\
& \left\|w(t)+\int_{t-\tau}^{t}(C A(u(s)) \hat{x}(s)+C b(u(s))) d s-y(t)\right\|^{2} \\
& \left.>\sigma(t) V_{y}\left(t-t_{e}+\tau\right)\right\}
\end{aligned}
$$

where $\sigma(t) \in \mathbb{R}$ is positive which is one of parameters designed/chosen by the user.

Remark 1: Actually, the above event-triggered rule (8) can be written in a simple form as $S_{e}=\left\{t \in S_{p} \mid\left\|w^{-}(t+\tau)-y(t)\right\|^{2}\right.$ $\left.>\sigma(t) V_{y}\left(t-t_{e}+\tau\right)\right\}$ without the integral part. However, we do not use this simple form because the inequality in (8) needs to be checked at each instant $t_{k}$, and we cannot have future value $w^{-}\left(t_{k}+\tau\right)$ at the current instant $t_{k}$, so we need to use the integral to make a prediction, in order to respect the causality, which is given in (8).

Actually, the event-triggered mechanism designed in this paper belongs to a static kind. In literature [37], [38], the dynamic eventtriggered mechanism is designed, where a filter is introduced to improve the original static one, resulting in a less restrictive trigger condition (less transmissions). This conception is useful to further improve the event-triggered mechanism designed in this paper.

\section{B. Convergence analysis}

Define the state observation error as $e=\hat{x}-x$, with equations (1) (4) and (6), one obtains the following dynamical equations:

$$
\left\{\begin{array}{l}
\dot{e}(t)=A(u) e(t) t \in\left[t_{k-1}+\tau, t_{k}+\tau\right) \\
e\left(t_{k}+\tau\right)=e_{1}\left(t_{k}+\tau\right)+e_{2}\left(t_{k}+\tau\right)
\end{array}\right.
$$

where $e_{1}\left(t_{k}+\tau\right)=e^{-}\left(t_{k}+\tau\right)-\rho t_{e} S^{-1}\left(t_{k}+\tau\right) C^{\prime} C e\left(t_{k}\right)$ and $e_{2}\left(t_{k}+\tau\right)=\rho t_{e} S^{-1}\left(t_{k}+\tau\right) C^{\prime}\left(w\left(t_{k}+\tau\right)-y\left(t_{k}\right)\right)$.

Consider the following Lyapunov function:

$$
V(t)=\frac{1}{4} e(t)^{\prime} S(t) e(t)
$$

where $S(t)$ is defined by (4) and (6) which is always symmetric positive definite, guaranteed by the following Proposition 1 . In this section, the goal is to prove the exponential decrease of Lyapunov function $V(t)$, including in continuous-time intervals and at discretetime instants. And at discrete-time instants, $V(t)$ should satisfy the condition $V\left(t_{k}+\tau\right) \leq \gamma\left(t_{k}\right) V\left(t_{k-1}+\tau\right)$ where $\gamma(t) \in \mathbb{R}$ satisfying $0<\gamma(t)<1$ for all $t=t_{k}, k \geq 1$. Both the parameters $\gamma(t)$ and $\sigma(t)$ can be designed later, and the specific method will be shown in Remark 3.

Then we can state the following result.

Proposition 1: Let $u$ be a regularly persistent input for system (1), for all $\mu \geq 2 \xi$ where $\xi=\sup _{t \geq t_{0}}\|A(u(t))\|$, if $t_{e} \leq \bar{T}$, where $\bar{T}$ is the unique positive term such that $\bar{T}=$ $\delta e^{-2 \xi\left(t_{a}+\bar{T}\right)} /\left(4 \xi\left\|C^{\prime} C\right\|\left(t_{a}+\bar{T}\right)\right)$, then, for all $S\left(t_{0}\right)$ symmetric positive definite, there exist constants $\alpha, \beta \in \mathbb{R}^{+}$such that, for $t \in\left[t_{0}, \infty\right)$

$$
\alpha I_{n} \leq S(t) \leq \beta I_{n}
$$

The proof of Proposition 1 can be found in [34].

Remark 2: $\xi$ is well defined because $A$ is continuous and the considered inputs belong to a compact set. $\bar{T}$ always exists: writing condition $\bar{T}=\delta e^{-2 \xi\left(t_{a}+\bar{T}\right)} /\left(4 \xi\left\|C^{\prime} C\right\|\left(t_{a}+\bar{T}\right)\right)$ as $4 \xi\left\|C^{\prime} C\right\|\left(t_{a}+\bar{T}\right) \bar{T}=\delta e^{-2 \xi\left(t_{a}+\bar{T}\right)}$, it is clear that for positive values of $\bar{T}$, the polynomial term on the left-hand side will cross the exponential on the right-hand side at a unique point.

Theorem 1: Assume that Hypothesis 1 holds and $\mu>2 \xi$ as stated in Proposition 1. The proposed observer (4-7) globally and exponentially converges to system (1) if there exist positive parameters $0<\gamma(t)<1, \sigma(t)>0$ for all $t=t_{k}, k \geq 1$ such that the following conditions are satisfied:

$\left\{\begin{array}{l}t_{e} \leq \min \left\{\frac{\alpha}{\rho\|C\|^{2}}, \bar{T}\right\} \\ e^{-\frac{\mu t_{e}}{4}}+\frac{4 \Delta_{5} \xi^{2}\|C\|^{2} \tau^{2}}{\alpha} e^{-\frac{\mu\left(t_{e}-\tau\right)}{4}}+\frac{4\left(\rho t_{e}\right)^{2}\|C\|^{4}}{\alpha^{2}} \sigma\left(t_{k}\right) \leq 2 \gamma\left(t_{k}\right)\end{array}\right.$

where $\Delta_{5}=\rho t_{e}\left(\left(1+\frac{\rho t_{e}}{\alpha}\|C\|^{2}\right)^{2}+\frac{\rho t_{e}}{\alpha}\|C\|^{2}\right)$.

Proof: Considering the Lyapunov function defined in (10), the proof of Theorem 1 can be divided into the following 3 steps. Step 1 proves the decrease of Lyapunov function in continuoustime intervals, and Step 2-3 proves the decrease of Lyapunov function at discrete-time instants. Specifically, Step 2 tries to prove $V\left(t_{k}+\tau\right)$ is bounded by a formula related to $V\left(t_{k-1}+\tau\right)$ and $\left\|w\left(t_{k}+\tau\right)-y\left(t_{k}\right)\right\|^{2}$, which will be used in Step 3 to prove that the Lyapunov function at discrete-time instants is decreasing if the event-triggered mechanism is designed as (8).

Step 1: To prove $V(t)$ is decreasing when $t \in\left[t_{k-1}+\tau, t_{k}+\tau\right)$.

Suppose that all conditions in (12) are satisfied. Then, with the considered Lyapunov function $V(t)$ for $t \in\left[t_{k-1}+\tau, t_{k}+\tau\right)$ defined in (10), its time derivative leads to

$$
\dot{V}(t)=-\frac{1}{4} \mu V(t)
$$

Step 2: To prove $V\left(t_{k}+\tau\right)$ is bounded by a formula related to $V\left(t_{k-1}+\tau\right)$ and $\left\|w\left(t_{k}+\tau\right)-y\left(t_{k}\right)\right\|^{2}$.

According to (9), compute $V\left(t_{k}+\tau\right)$ as

$$
\begin{aligned}
V\left(t_{k}+\tau\right)= & \frac{1}{4} e\left(t_{k}+\tau\right)^{\prime} S\left(t_{k}+\tau\right) e\left(t_{k}+\tau\right) \\
= & \frac{1}{4}\left(e_{1}\left(t_{k}+\tau\right)+e_{2}\left(t_{k}+\tau\right)\right)^{\prime} S\left(t_{k}+\tau\right) \\
& \left(e_{1}\left(t_{k}+\tau\right)+e_{2}\left(t_{k}+\tau\right)\right) \\
\leq & \frac{1}{2}\left(e_{1}\left(t_{k}+\tau\right)^{\prime} S\left(t_{k}+\tau\right) e_{1}\left(t_{k}+\tau\right)\right. \\
& \left.+e_{2}\left(t_{k}+\tau\right)^{\prime} S\left(t_{k}+\tau\right) e_{2}\left(t_{k}+\tau\right)\right)
\end{aligned}
$$

where we applied the Young's inequality.

First, let us deal with $e_{1}\left(t_{k}+\tau\right)^{\prime} S\left(t_{k}+\tau\right) e_{1}\left(t_{k}+\tau\right)$. By using the Leibniz integration formula, one obtains

$$
e^{-}\left(t_{k}+\tau\right)=e\left(t_{k}\right)+\int_{t_{k}}^{t_{k}+\tau} \dot{e}(s) d s
$$


By substituting $e\left(t_{k}\right)$ with (15), $e_{1}\left(t_{k}+\tau\right)$ can be written as

$$
\begin{aligned}
e_{1}\left(t_{k}+\tau\right)= & e^{-}\left(t_{k}+\tau\right)-\rho t_{e} S^{-1}\left(t_{k}+\tau\right) C^{\prime} C \\
& e^{-}\left(t_{k}+\tau\right)+\rho t_{e} S^{-1}\left(t_{k}+\tau\right) \\
& C^{\prime} C \int_{t_{k}}^{t_{k}+\tau} \dot{e}(s) d s \\
= & e^{-}\left(t_{k}+\tau\right)-\rho t_{e} S^{-1}\left(t_{k}+\tau\right) C^{\prime} C \\
& e^{-}\left(t_{k}+\tau\right)+\rho t_{e} S^{-1}\left(t_{k}+\tau\right) \\
& C^{\prime} C \int_{t_{k}}^{t_{k}+\tau} A(u(s)) e(s) d s
\end{aligned}
$$

Compute $e_{1}\left(t_{k}+\tau\right)^{\prime} S\left(t_{k}+\tau\right) e_{1}\left(t_{k}+\tau\right)$ according to (16) as

$$
\begin{aligned}
& e_{1}\left(t_{k}+\tau\right)^{\prime} S\left(t_{k}+\tau\right) e_{1}\left(t_{k}+\tau\right) \\
& =e^{-}\left(t_{k}+\tau\right)^{\prime} \Delta_{1}\left(t_{k}+\tau\right) e^{-}\left(t_{k}+\tau\right) \\
& \quad+e^{-}\left(t_{k}+\tau\right)^{\prime} \Delta_{2}\left(t_{k}+\tau\right) I_{0}+I_{0}^{\prime} \Delta_{3}\left(t_{k}+\tau\right) I_{0}
\end{aligned}
$$

where

$$
\begin{aligned}
\Delta_{1}\left(t_{k}+\tau\right)= & S\left(t_{k}+\tau\right)-2 \rho t_{e} C^{\prime} C \\
& +\rho^{2} t e^{2} C^{\prime} C S^{-1}\left(t_{k}+\tau\right) C^{\prime} C \\
\Delta_{2}\left(t_{k}+\tau\right)= & 2 \rho t_{e}\left(I_{n}-\rho t_{e} C^{\prime} C S^{-1}\left(t_{k}+\tau\right)\right) C^{\prime} C \\
\Delta_{3}\left(t_{k}+\tau\right)= & \rho^{2} t_{e}^{2} C^{\prime} C S^{-1}\left(t_{k}+\tau\right) C^{\prime} C \\
I_{0}= & \int_{t_{k}}^{t_{k}+\tau} A(u(s)) e(s) d s
\end{aligned}
$$

With some further computations, one gets

$$
\begin{aligned}
e^{-} & \left(t_{k}+\tau\right)^{\prime} \Delta_{1}\left(t_{k}+\tau\right) e^{-}\left(t_{k}+\tau\right) \\
= & V^{-}\left(t_{k}+\tau\right)+\rho^{2} t_{e}^{2} e_{y}^{-}\left(t_{k}+\tau\right)^{\prime} C S^{-1}\left(t_{k}+\tau\right) \\
& C^{\prime} e_{y}^{-}\left(t_{k}+\tau\right)-2 \rho t_{e}\left\|e_{y}^{-}\left(t_{k}+\tau\right)\right\|^{2} \\
\leq & V^{-}\left(t_{k}+\tau\right)+\rho^{2} t_{e}^{2} \alpha^{-1}\|C\|^{2}\left\|e_{y}^{-}\left(t_{k}+\tau\right)\right\|^{2} \\
& \quad-2 \rho t_{e}\left\|e_{y}^{-}\left(t_{k}+\tau\right)\right\|^{2}
\end{aligned}
$$

and

$$
\begin{aligned}
& e^{-}\left(t_{k}+\tau\right)^{\prime} \Delta_{2}\left(t_{k}+\tau\right) I_{0} \\
& =2 \rho t_{e} e^{-}\left(t_{k}+\tau\right)^{\prime} C^{\prime}\left(I_{p}-\rho t_{e} C S^{-1}\left(t_{k}+\tau\right) C^{\prime}\right) C I_{0} \\
& \leq 2 \rho t_{e}\left(1+\frac{\rho t_{e}}{\alpha}\|C\|^{2}\right)\left\|C I_{0}\right\|\left\|e_{y}^{-}\left(t_{k}+\tau\right)\right\| \\
& \leq \rho t_{e}\left(1+\frac{\rho t_{e}}{\alpha}\|C\|^{2}\right)^{2}\left\|C I_{0}\right\|^{2}+\rho t_{e}\left\|e_{y}^{-}\left(t_{k}+\tau\right)\right\|^{2}
\end{aligned}
$$

From equations (17-20), one obtains

$$
\begin{aligned}
& e_{1}\left(t_{k}+\tau\right)^{\prime} S\left(t_{k}+\tau\right) e_{1}\left(t_{k}+\tau\right) \\
& \leq V^{-}\left(t_{k}+\tau\right)+t_{e} \Delta_{4}\left\|e_{y}^{-}\left(t_{k}+\tau\right)\right\|^{2}+\Delta_{5}\left\|C I_{0}\right\|^{2}
\end{aligned}
$$

with

$$
\begin{aligned}
& \Delta_{4}=\frac{\rho^{2} t_{e}\|C\|^{2}}{\alpha}-\rho \\
& \Delta_{5}=\rho t_{e}\left(\left(1+\frac{\rho t_{e}}{\alpha}\|C\|^{2}\right)^{2}+\frac{\rho t_{e}}{\alpha}\|C\|^{2}\right)
\end{aligned}
$$

The condition $\Delta_{4} \leq 0$ is satisfied with

$$
t_{e} \leq \frac{\alpha}{\rho\|C\|^{2}}
$$

From (13), one can get

$$
V(t)=V\left(t_{k-1}+\tau\right) e^{-\frac{1}{4} \mu\left(t-t_{k-1}-\tau\right)} \leq V\left(t_{k-1}+\tau\right)
$$

on $\left[t_{k-1}+\tau, t_{k}+\tau\right)$. With (11) and (24), one obtains

$$
\frac{\alpha}{4}\|e(t)\|^{2} \leq V(t) \leq V\left(t_{k-1}+\tau\right)
$$

on $\left[t_{k-1}+\tau, t_{k}+\tau\right)$.

According to (18), one can get

$$
\begin{aligned}
\left\|C I_{0}\right\| & =\left\|C \int_{t_{k}}^{t_{k}+\tau} A(u(s)) e(s) d s\right\| \\
& \leq\|C\| \int_{t_{k}}^{t_{k}+\tau}\|A(u(s))\|\|e(s)\| d s
\end{aligned}
$$

Similar to the inequality (25), we have $\frac{\alpha}{4}\|e(t)\|^{2} \leq V(t) \leq$ $e^{-\frac{1}{4} \mu\left(t_{e}-\tau\right)} V\left(t_{k-1}+\tau\right)$ on $\left[t_{k}, t_{k}+\tau\right)$, and with $\|A(u)\| \leq \bar{\xi}$, (26) yields

$$
\begin{aligned}
\left\|C I_{0}\right\|^{2} & \leq\|C\|^{2} \xi^{2}\left(\int_{t_{k}}^{t_{k}+\tau}\|e(s)\| d s\right)^{2} \\
& \leq \frac{4\|C\|^{2} \xi^{2}}{\alpha} \tau^{2} e^{-\frac{1}{4} \mu\left(t_{e}-\tau\right)} V\left(t_{k-1}+\tau\right)
\end{aligned}
$$

With (23-24) and (27), the inequality (21) can be further deduced as

$$
\begin{aligned}
& e_{1}\left(t_{k}+\tau\right)^{\prime} S\left(t_{k}+\tau\right) e_{1}\left(t_{k}+\tau\right) \\
& \leq\left(e^{-\frac{1}{4} \mu t_{e}}+\Delta_{5} \frac{4 \xi^{2}\|C\|^{2}}{\alpha} \tau^{2} e^{-\frac{1}{4} \mu\left(t_{e}-\tau\right)}\right) V\left(t_{k-1}+\tau\right)
\end{aligned}
$$

After dealing with $e_{1}\left(t_{k}+\tau\right)^{\prime} S\left(t_{k}+\tau\right) e_{1}\left(t_{k}+\tau\right)$, we can then analyze $e_{2}\left(t_{k}+\tau\right)^{\prime} S\left(t_{k}+\tau\right) e_{2}\left(t_{k}+\tau\right)$, with (11), which can be written as

$$
\begin{aligned}
& e_{2}\left(t_{k}+\tau\right)^{\prime} S\left(t_{k}+\tau\right) e_{2}\left(t_{k}+\tau\right) \\
&=\left(\rho t_{e} S^{-1}\left(t_{k}+\tau\right) C^{\prime}\left(w\left(t_{k}+\tau\right)-y\left(t_{k}\right)\right)\right)^{\prime} S\left(t_{k}+\tau\right) \\
& \quad\left(\rho t_{e} S^{-1}\left(t_{k}+\tau\right) C^{\prime}\left(w\left(t_{k}+\tau\right)-y\left(t_{k}\right)\right)\right) \\
& \leq \frac{\left(\rho t_{e}\right)^{2}}{\alpha}\|C\|^{2}\left\|w\left(t_{k}+\tau\right)-y\left(t_{k}\right)\right\|^{2}
\end{aligned}
$$

Then, gather the result stated in (28) for $e_{1}\left(t_{k}+\tau\right)^{\prime} S\left(t_{k}+\right.$ $\tau) e_{1}\left(t_{k}+\tau\right)$ and the result stated in (29) for $e_{2}\left(t_{k}+\tau\right)^{\prime} S\left(t_{k}+\right.$ $\tau) e_{2}\left(t_{k}+\tau\right)$, and with (14), one has

$$
\begin{aligned}
& V\left(t_{k}+\tau\right) \\
& \leq \frac{1}{2}\left(e^{-\frac{1}{4} \mu t_{e}}+\Delta_{5} \frac{4 \xi^{2}\|C\|^{2}}{\alpha} \tau^{2} e^{-\frac{1}{4} \mu\left(t_{e}-\tau\right)}\right) V\left(t_{k-1}+\tau\right) \\
& \quad+\frac{1}{2} \frac{\left(\rho t_{e}\right)^{2}}{\alpha}\|C\|^{2}\left\|w\left(t_{k}+\tau\right)-y\left(t_{k}\right)\right\|^{2}
\end{aligned}
$$

Step 3: To prove $V\left(t_{k}+\tau\right), k \in \mathbb{N}$, is decreasing if the eventtriggered mechanism is designed as (8).

It is clear that, if there exists a positive parameter $0<\gamma(t)<1$, such that

$$
V\left(t_{k}+\tau\right) \leq \gamma\left(t_{k}\right) V\left(t_{k-1}+\tau\right)
$$

then $V\left(t_{k}+\tau\right)$ for $k \geq 1$ is decreasing, which is a sufficient condition to prove the convergence of the proposed observer with (13). According to (30), it can be checked that the inequality (31) is satisfied if

$$
\begin{aligned}
& \frac{1}{2}\left(e^{-\frac{1}{4} \mu t_{e}}+\Delta_{5} \frac{4 \xi^{2}\|C\|^{2}}{\alpha} \tau^{2} e^{-\frac{1}{4} \mu\left(t_{e}-\tau\right)}\right) V\left(t_{k-1}+\tau\right) \\
& +\frac{1}{2} \frac{\left(\rho t_{e}\right)^{2}}{\alpha}\|C\|^{2}\left\|w\left(t_{k}+\tau\right)-y\left(t_{k}\right)\right\|^{2} \leq \gamma\left(t_{k}\right) V\left(t_{k-1}+\tau\right)
\end{aligned}
$$


is satisfied. The inequality (32) can be further rewritten as

$$
\begin{aligned}
& \left\|w\left(t_{k}+\tau\right)-y\left(t_{k}\right)\right\|^{2} \\
\leq & \left(2 \gamma\left(t_{k}\right)-e^{-\frac{1}{4} \mu t_{e}}-\Delta_{5} \frac{4 \xi^{2}\|C\|^{2}}{\alpha} \tau^{2} e^{-\frac{1}{4} \mu\left(t_{e}-\tau\right)}\right) \\
& \frac{\alpha}{\left(\rho t_{e}\right)^{2}\|C\|^{2}} V\left(t_{k-1}+\tau\right)
\end{aligned}
$$

Because $V\left(t_{k-1}+\tau\right)$ is unknown in the detection of the eventtriggered condition, with (11), we have the following transformation between $V\left(t_{k-1}+\tau\right)$ and $V_{y}\left(t_{k-1}+\tau\right)$

$$
\begin{aligned}
V_{y}\left(t_{k-1}+\tau\right) & =e_{y}^{\prime}\left(t_{k-1}+\tau\right) e_{y}\left(t_{k-1}+\tau\right) \\
& =e^{\prime}\left(t_{k-1}+\tau\right) C^{\prime} C e\left(t_{k-1}+\tau\right) \\
& \leq \frac{4\|C\|^{2}}{\alpha} \frac{1}{4} \alpha\left\|e\left(t_{k-1}+\tau\right)\right\|^{2} \\
& \leq \frac{4\|C\|^{2}}{\alpha} V\left(t_{k-1}+\tau\right)
\end{aligned}
$$

According to (34), the inequality (33) is true if

$$
\begin{aligned}
& \left\|w\left(t_{k}+\tau\right)-y\left(t_{k}\right)\right\|^{2} \\
\leq & \left(2 \gamma\left(t_{k}\right)-e^{-\frac{1}{4} \mu t_{e}}-\Delta_{5} \frac{4 \xi^{2}\|C\|^{2}}{\alpha} \tau^{2} e^{-\frac{1}{4} \mu\left(t_{e}-\tau\right)}\right) \\
& \frac{\alpha^{2}}{4\left(\rho t_{e}\right)^{2}\|C\|^{4}} V_{y}\left(t_{k-1}+\tau\right)
\end{aligned}
$$

is satisfied. And the inequality (35) is satisfied if the following inequality is satisfied

$$
\left\|w\left(t_{k}+\tau\right)-y\left(t_{k}\right)\right\|^{2} \leq \sigma\left(t_{k}\right) V_{y}\left(t_{k-1}+\tau\right)
$$

where

$$
\begin{aligned}
& \sigma\left(t_{k}\right) \\
& \leq\left(2 \gamma\left(t_{k}\right)-e^{-\frac{1}{4} \mu t_{e}}-\Delta_{5} \frac{4 \xi^{2}\|C\|^{2}}{\alpha} \tau^{2} e^{-\frac{1}{4} \mu\left(t_{e}-\tau\right)}\right) \frac{\alpha^{2}}{4\left(\rho t_{e}\right)^{2}\|C\|^{4}}
\end{aligned}
$$

which actually equals to the second condition of (12). And according to it, one can choose both the parameters $\gamma(t)$ and $\sigma(t)$.

According to (5), one has

$$
\begin{aligned}
w^{-}\left(t_{k}+\tau\right)= & w\left(t_{k}\right)+\int_{t_{k}}^{t_{k}+\tau} \dot{w}(t) d t \\
= & w\left(t_{k}\right)+\int_{t_{k}}^{t_{k}+\tau}(C A(u(t-\tau)) \hat{x}(t-\tau) \\
& +C b(u(t-\tau))) d t \\
= & w\left(t_{k}\right)+\int_{t_{k}-\tau}^{t_{k}}(C A(u(t)) \hat{x}(t)+C b(u(t))) d t
\end{aligned}
$$

Actually, the inequality (36) is always true because of the following statements:

Case 1: If the inequality in (8) is satisfied at the instant $t=t_{k}$, the transmission of the output data will be performed, which means $\exists j \geq 0, \bar{t}_{j}=t_{k}$ and the value of $w$ is updated with the real system's output as $w\left(\bar{t}_{j}+\tau\right)=y\left(\bar{t}_{j}\right)$, so the inequality (36) is satisfied since $\left\|w\left(\bar{t}_{j}+\tau\right)-y\left(\bar{t}_{j}\right)\right\|^{2}=0$, which means $\left\|w\left(t_{k}+\tau\right)-y\left(t_{k}\right)\right\|^{2}=0$.

Case 2: If the inequality in (8) is not satisfied at the instant $t=t_{k}$, i.e., $\left\|w\left(t_{k}\right)+\int_{t_{k}-\tau}^{t_{k}}(C A(u(t)) \hat{x}(t)+C b(u(t))) d t-y\left(t_{k}\right)\right\|^{2} \leq$ $\sigma\left(t_{k}\right) V_{y}\left(t_{k-1}+\tau\right)$, which equals to $\left\|w^{-}\left(t_{k}+\tau\right)-y\left(t_{k}\right)\right\|^{2} \leq$ $\sigma\left(t_{k}\right) V_{y}\left(t_{k-1}+\tau\right)$, then the transmission will not be carried out, so $w$ is not updated with the real output data, and one has $w\left(t_{k}+\tau\right)=$ $w^{-}\left(t_{k}+\tau\right)$, therefore the inequality (36) is also satisfied.

Finally, we can prove that, if the event-triggered mechanism is designed as (8), with (37), the inequality in (36) is always satisfied for $\forall k \geq 1$, which means (31) is satisfied. The conditions (31) and (13) imply that the proposed observer (4-7) globally and exponentially converges to system (1).

Remark 3: The second inequality of (12) $e^{-\frac{\mu t_{e}}{4}}+$ $\frac{4 \Delta_{5} \xi^{2}\|C\|^{2} \tau^{2}}{\alpha} e^{-\frac{\mu\left(t_{e}-\tau\right)}{4}}+\frac{4\left(\rho t_{e}\right)^{2}\|C\|^{4}}{\alpha^{2}} \sigma\left(t_{k}\right) \leq 2 \gamma\left(t_{k}\right)$ makes it possible for users to select both the parameters $\gamma(t)$ and $\sigma(t)$ with $t=t_{k} \in S_{p}$. In the practical applications, one can first choose the parameter $\gamma(t)$ with $t=t_{k} \in S_{p}$ in the premise of $0<\gamma\left(t_{k}\right)<1$ and $2 \gamma\left(t_{k}\right)-e^{-\frac{\mu t_{e}}{4}}-\frac{4 \Delta_{5} \xi^{2}\|C\|^{2} \tau^{2}}{\alpha} e^{-\frac{\mu\left(t_{e}-\tau\right)}{4}}>0$ (the smaller value of $\gamma(t)$ with $t=t_{k} \in S_{p}$ implies the faster convergence of the proposed observer). Under this selected value of $\gamma(t)$ with $t=t_{k} \in S_{p}$, the existence of positive $\sigma(t)$ with $t=t_{k} \in S_{p}$ is guaranteed. Thus, one can select the positive value of $\sigma(t)$ with $t=t_{k} \in S_{p}$ according to the second condition of (12). The smaller value of $\sigma(t)$ with $t=t_{k} \in S_{p}$ implies more triggered events, i.e., more information needs to be sent to the observer. Actually, the choice of those two parameters $\gamma(t)$ and $\sigma(t)$ with $t=t_{k} \in S_{p}$ is a compromise between the performance and the economy. We would like to highlight the parameters $\gamma(t)$ and $\sigma(t)$ with $t=t_{k} \in S_{p}$ are time-varying. For simplifying the design, we can also choose a uniform constant, which means $\gamma(t)=\gamma$ and $\sigma(t)=\sigma$ for all $t=t_{k} \in S_{p}$.

\section{Comments}

In this section, we would like to highlight some advantages of the proposed observer over the existing results in the literature, especially compared with the result of [33].

Firstly, the Lyapunov function is chosen as $V(t)=\eta e(t)^{\prime} S(t) e(t)$ with $\eta \in \mathbb{R}$ satisfying $\eta>0$, so that one can choose the coefficient $\eta$ of Lyapunov function, and besides the last step of the deduction in inequality (20) is changed to $e^{-}\left(t_{k}+\tau\right)^{\prime} \Delta_{2}\left(t_{k}+\tau\right) I_{0} \leq$ $\chi \rho t_{e}\left(1+\frac{\rho t_{e}}{\alpha}\|C\|^{2}\right)^{2}\left\|C I_{0}\right\|^{2}+\frac{1}{\chi} \rho t_{e}\left\|e_{y}^{-}\left(t_{k}+\tau\right)\right\|^{2}$ with $\chi \in \mathbb{R}$ satisfying $\chi>0$ so that we can choose the coefficient $\chi$, and finally make the sending instant being periodic $\left(t_{e}\right)$, i.e. we have $S_{e}=S_{p}$, thus we will have no $\sigma(t)$ in the condition (38). Therefore, the following theoretical conditions of periodic case is obtained (Since $\gamma(t)$ for all $t$ is set to be infinitely close to 1 in [33], we have the same setting here for the comparison):

$$
\left\{\begin{array}{l}
t_{e} \leq \min \left\{\frac{\alpha}{\rho\|C\|^{2}}\left(2-\frac{1}{\chi}\right), \bar{T}\right\} \\
\eta e^{-\eta \mu t_{e}}+\Delta_{5}(\chi) \frac{\xi^{2}\|C\|^{2}}{\alpha} \tau^{2} e^{-\eta \mu\left(t_{e}-\tau\right)}<1 \\
\tau \leq t_{e}
\end{array}\right.
$$

with $\Delta_{5}(\chi)=\rho t_{e}\left(\chi\left(1+\frac{\rho t_{e}}{\alpha}\|C\|^{2}\right)^{2}+\frac{\rho t_{e}}{\alpha}\|C\|^{2}\right)$.

Note that the theoretical conditions obtained in [33] with periodic transmissions are as follows:

$$
\left\{\begin{array}{l}
t_{e} \leq \min \left\{\frac{\alpha\left(\frac{3}{2} \rho-1\right)}{\rho^{2}\|C\|^{2}}, \bar{T}\right\} \\
e^{-\mu t_{e}}+\Delta_{5}(2) \frac{\xi^{2}\|C\|^{2}}{\alpha} \tau^{2}<1 \\
\tau \leq t_{e}
\end{array}\right.
$$

with the same value of $\Delta_{5}(\chi)$ as that in (38). The following remarks can be made.

Remark 4: When the constraints of transmission period $t_{e}$ are the same in (38) and (39), the proposed observer in this paper has less restrictive constraints of delay $\tau$. In fact, One can obtain $\chi=\frac{2 \rho}{2+\rho}$ when $\frac{\alpha}{\rho\|C\|^{2}}\left(2-\frac{1}{\chi}\right)=\frac{\alpha\left(\frac{3}{2} \rho-1\right)}{\rho^{2}\|C\|^{2}}$ is satisfied. Thus, the proposed 
method is less restrictive in the sense of delay because the following inequalities are satisfied:

$$
\left\{\begin{array}{l}
\eta e^{-\eta \mu t_{e}} \leq e^{-\mu t_{e}}, \exists \eta \\
\Delta_{5}\left(\frac{2 \rho}{2+\rho}\right) \leq \Delta_{5}(2), \rho \geq 1 \\
e^{-\eta \mu\left(t_{e}-\tau\right)}<1
\end{array}\right.
$$

Remark 5: When the constraints of delay $\tau$ are the same in (38) and (39), the proposed method is also less restrictive in the sense of transmission period $t_{e}$. The proof is easy to get with the similar analysis.

\section{SiMULATIONS}

In this section, a numerical example is considered to illustrate the effectiveness of the proposed event-triggered observer.

\section{A. Simulation results}

A second-order system from [39] is considered as follows

$$
\left\{\begin{array}{l}
\dot{x}_{1}=x_{2} \sin u \\
\dot{x}_{2}=-0.1 x_{1} \sin u-0.2 x_{2} \\
y=x_{1}
\end{array}\right.
$$

where $u(t)=2-\cos ^{2}(0.1 t)$. According to theoretical conditions stated in Section III, after some calculations, the detection period of event-triggered condition can be set as $t_{e}=0.11 \mathrm{~s}$. The upper bound of unknown transmission delays can be set as $\tau=0.5 t_{e}=0.055 \mathrm{~s}$. An event-triggered observer is designed of the form (4-7) for system (41). The relevant design parameters of event-triggered observer are set as $\rho=1$ and $\mu=2.1$. The initial conditions are set as $t_{0}=0, x_{1}(0)=x_{2}(0)=50, \hat{x}_{1}(0)=\hat{x}_{2}(0)=0$ and $S_{0}=\left[\begin{array}{ll}0.23 & 0 \\ 0 & 0.46\end{array}\right]$. By using numerical tools, the following values can be found $\delta=0.5, t_{a}=0.01,2 \xi=2.0396$ and $\alpha \geq 0.1125$. Thus, the first condition in (12) is satisfied, i.e. $t_{e} \leq \min \{0.1125,0.21\}$. We choose two groups of values for $\gamma$ according to the rule in Remark 3, including:

(a) $\gamma$ is a constant: $\gamma=0.6,0.7,0.9,0.93$.

(b) $\gamma$ is time-varying:

$$
\begin{aligned}
& \gamma_{0}(t)= \begin{cases}\sin (0.05 t+1) & t \in[0,10] \\
\sin (1.5)=0.9975 & t>10\end{cases} \\
& \gamma_{1}(t)= \begin{cases}\sin (0.05 t+0.8) & t \in[0,10] \\
\sin (1.3)=0.96 & t>10\end{cases} \\
& \gamma_{2}(t)= \begin{cases}0.85 \sin (0.05 t+0.8) & t \in[0,10] \\
0.85 \sin (1.3)=0.82 & t>10\end{cases} \\
& \gamma_{3}(t)= \begin{cases}0.7 \sin (0.05 t+0.8) & t \in[0,10] \\
0.7 \sin (1.3)=0.67 & t>10\end{cases}
\end{aligned}
$$

Thus, with the above settings, the maximum value of parameter $\sigma\left(t_{k}\right)$ of event-triggered mechanism can be calculated according to the second condition in (12) as:

(a) $\sigma=0.0517,0.1040,0.2086,0.2243$.

(b) $\sigma(t)=(\gamma(t)-0.5012) / 1.9121, t \geq 0$
Consequently, according to Theorem 1, the event-triggered observer is stable under the above parameter settings. Fig. 3 and 4 show the estimation results and event-triggered instants, respectively under the first group of values of $\gamma$. Fig. 5, 6, and 7 show the dynamics of $\gamma$, estimation results, and event-triggered instants, respectively under the second group of values of $\gamma$. From those figures, one can see that the good estimation performance is obtained with very few data transmissions by using the designed method in this paper. Besides, when $\gamma$ is smaller, faster convergence rate of estimation error and more bandwidth occupation will be obtained. So, one can adjust the parameter $\gamma$ to obtain a trade-off between the estimation performance and bandwidth savings.
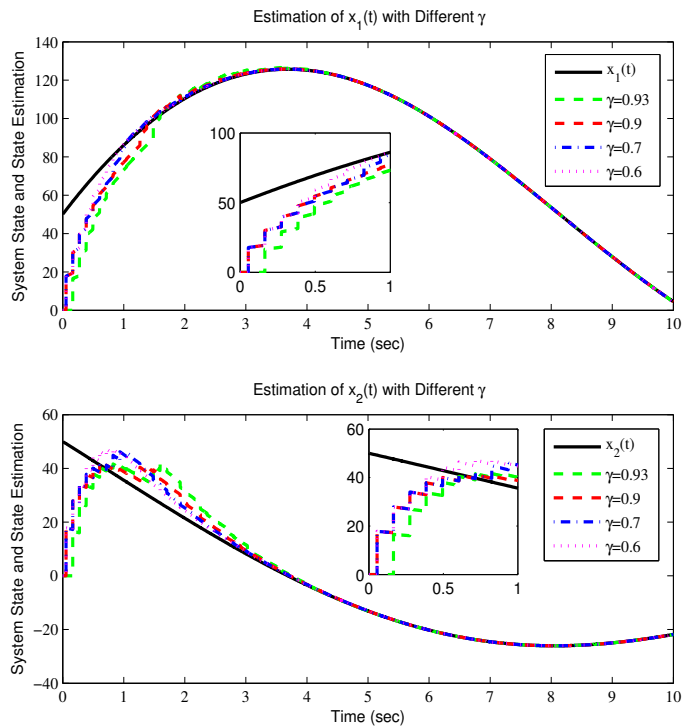

Fig. 3. State estimation when $\gamma$ is constant (group(a))
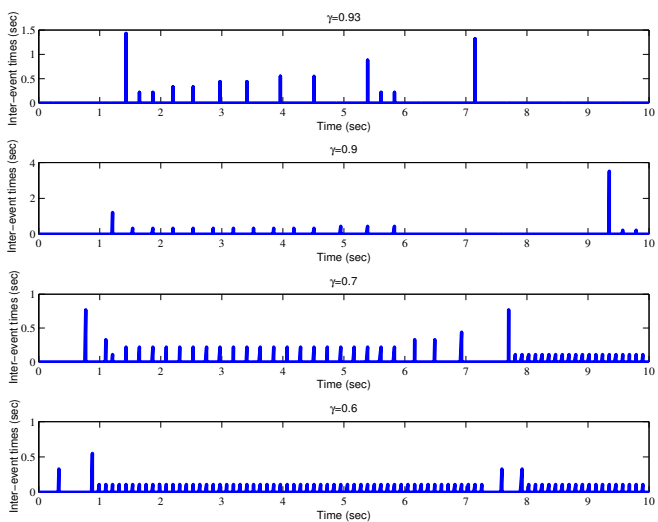

Fig. 4. Inter-event times when $\gamma$ is constant (group(a))

\section{B. Comparison with the method of [33]}

In this section, the event-triggered observer method proposed in this paper and the method stated in [33] will be compared in simulations.

In the method of [33], the sampling period is $0.06 \mathrm{~s}$ which satisfies the theoretical condition. The setting of other parameters is same as that in Section V-A, and the relevant simulation results will be 


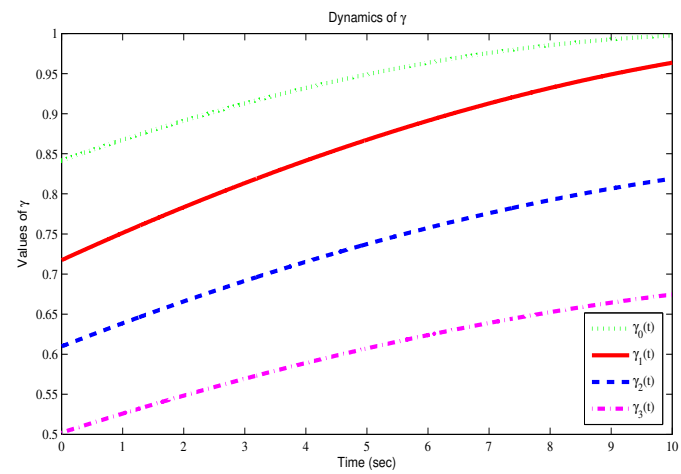

Fig. 5. Dynamics of $\gamma$ (group(b))
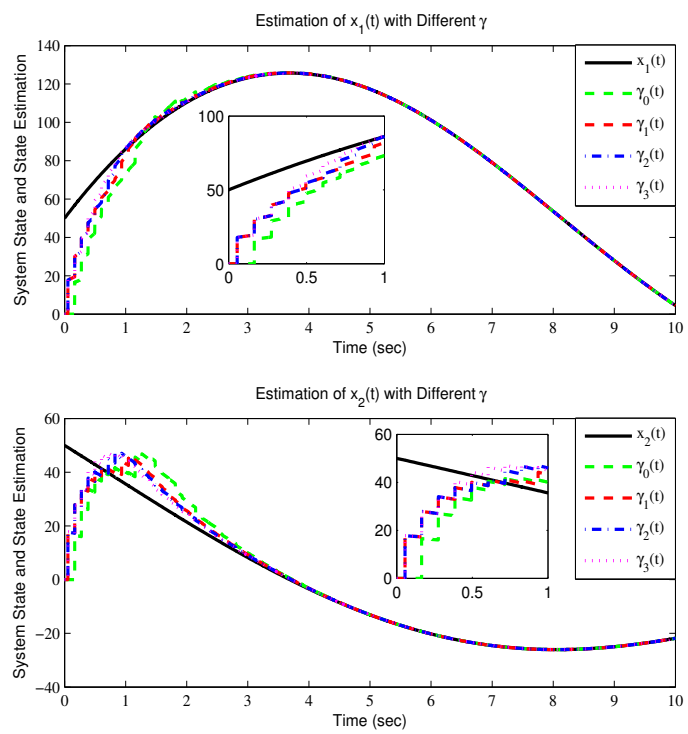

Fig. 6. State estimation when $\gamma$ is time-varying (group(b))

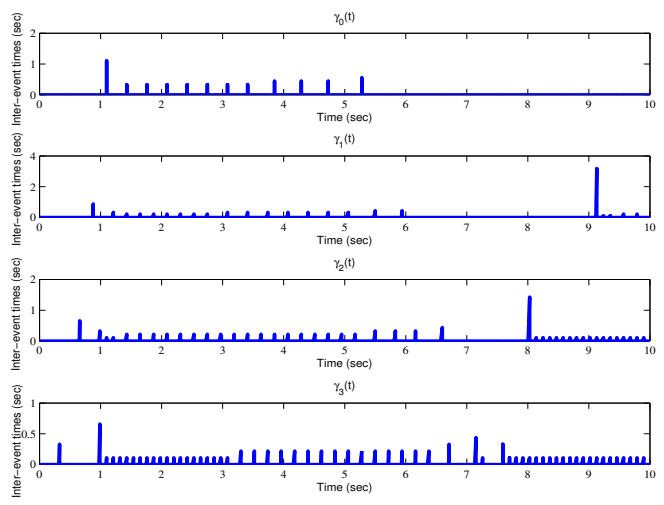

Fig. 7. Inter-event times when $\gamma$ is time-varying (group(b))

compared with the results based on $\gamma_{3}$ of group $(b)$ defined in Section V-A.

Fig. 8 shows the estimation results of two methods, and Table I shows the number of transmissions in 10 s for these two methods. According to Fig. 8 and Table I, one can see that, compared with the method in [33], the number of transmissions when applying
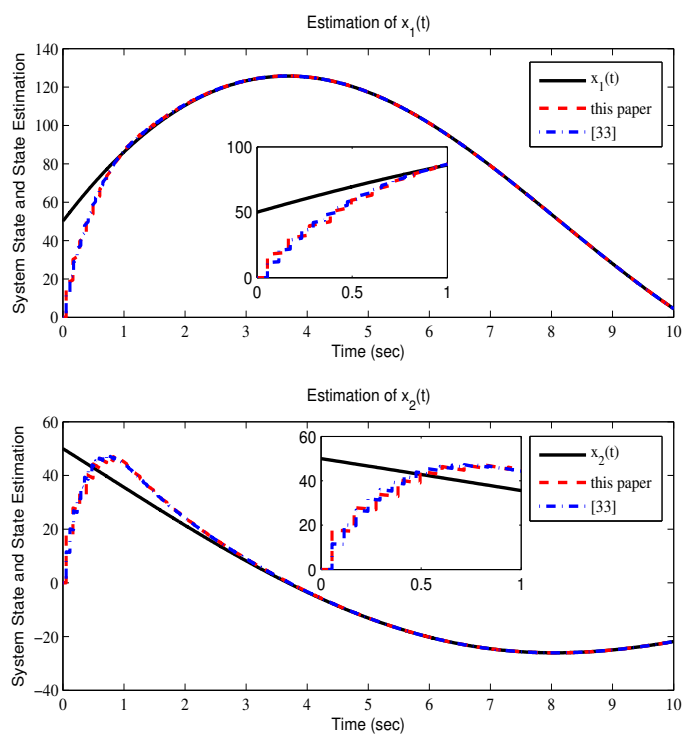

Fig. 8. State estimation in different methods

the proposed observer of this paper is reduced while the estimation performance is almost the same for these two methods.

TABLE I

NUMBER OF TRANSMISSIONS IN $10 s$ BASED ON DIFFERENT METHODS.

\begin{tabular}{ccc}
\hline Methods & This paper & {$[33]$} \\
\hline Number of transmissions & 62 & 166 \\
\hline
\end{tabular}

\section{CONCLUSION}

An event-triggered observer for nonlinear state affine systems with delayed and sampled output is designed in this paper. The effectiveness of the designed observer is illustrated by simulations. The bandwidth occupation is reduced while the comparable estimation performance is guaranteed. The current work is based on a particular nonlinear system, which may be generalized to general nonlinear systems in future studies. Besides, the novel event-triggered mechanism designed in this paper can be further improved by introducing a filter to design a dynamic one.

\section{REFERENCES}

[1] A. Y. Lu, D. Zhai, J. Dong, Q. L. Zhang, A. Y. Lu, D. Zhai, J. Dong, and Q. L. Zhang, "Network-based fuzzy $H_{\infty}$ controller design for T$\mathrm{S}$ fuzzy systems via a new event-triggered communication scheme," Neurocomputing, 2017.

[2] F. D. Brunner, W. P. M. H. Heemels, and F. Allgower, "Robust eventtriggered MPC with guaranteed asymptotic bound and average sampling rate," IEEE Transactions on Automatic Control, vol. PP, no. 99, pp. 1-1, 2017.

[3] B. Demirel, V. Gupta, D. E. Quevedo, and M. Johansson, "On the tradeoff between communication and control cost in event-triggered dead-beat control," IEEE Transactions on Automatic Control, vol. PP, no. 99, pp. $1-1,2017$.

[4] L. Li, X. Wang, and M. D. Lemmon, "Efficiently attentive eventtriggered systems with limited bandwidth," IEEE Transactions on Automatic Control, vol. 62, no. 3, pp. 1491-1497, 2017.

[5] L. Xing, C. Wen, Z. Liu, H. Su, and J. Cai, "Event-triggered adaptive control for a class of uncertain nonlinear systems," IEEE Transactions on Automatic Control, vol. 62, no. 4, pp. 2071-2076, 2017. 
[6] D. Silvestre, P. Rosa, J. P. Hespanha, and C. Silvestre, "Self-triggered and event-triggered set-valued observers," Information Sciences, no. 0, 2017.

[7] B. A. Khashooei, D. J. Antunes, and W. P. M. H. Heemels, "Outputbased event-triggered control with performance guarantees," IEEE Transactions on Automatic Control, vol. PP, no. 99, pp. 1-1, 2017.

[8] A. Tanwani, C. Prieur, and M. Fiacchini, "Observer-based feedback stabilization of linear systems with event-triggered sampling and dynamic quantization ," Systems and Control Letters, vol. 94, pp. 46-56, 2016.

[9] H. P. Wang, Y. Tian, and N. Christov, "Event-triggered observer based control of networked visual servoing control systems," Control Engineering and Applied Informatics, vol. 16, no. 1, pp. 22-30, 2014.

[10] A. Y. Lu and G. H. Yang, "Event-triggered secure observer-based control for cyber-physical systems under adversarial attacks," Information Sciences, vol. 420, 2017.

[11] D. P. Borgers, V. S. Dolk, and W. P. M. H. Heemels, "Riccati-based design of event-triggered controllers for linear systems with delays,' IEEE Transactions on Automatic Control, vol. PP, no. 99, pp. 1-1, 2017.

[12] C. Song, H. Wang, Y. Tian, and N. Christov, "Event-triggered PCS based trajectory tracking control of systems with perturbation," Asian Journal of Control, vol. 19, no. 3, 2017.

[13] Y. Shoukry and P. Tabuada, "Event-triggered state observers for sparse sensor noise/attacks," IEEE Transactions on Automatic Control, vol. 61, no. 8, pp. 2079-2091, 2016.

[14] L. Etienne, S. Digennaro, and J. P. Barbot, "Periodic event-triggered observation and control for nonlinear lipschitz systems using impulsive observers," International Journal of Robust and Nonlinear Control, no. 4, 2017.

[15] H. Yuan, J. Wang, D. Shi, and S. Ling, "Towards event-triggered extended state observer," IEEE Transactions on Automatic Control, vol. 63, no. 6, pp. 1842-1849, 2018.

[16] C. Peng, S. Ma, and X. Xie, "Observer-based Non-PDC control for networked T-S fuzzy systems with an event-triggered communication." IEEE Transactions on Cybernetics, vol. PP, no. 99, pp. 1-9, 2017.

[17] Z. Zhong and Y. Zhu, "Observer-based output-feedback control of largescale networked fuzzy systems with two-channel event-triggering ," Journal of the Franklin Institute, 2017.

[18] M. Abdelrahim, R. Postoyan, J. Daafouz, and D. Nešić, "Stabilization of nonlinear systems using event-triggered output feedback controllers," IEEE Transactions on Automatic Control, vol. 61, no. 9, pp. 2682-2687, 2016.

[19] A. Tanwani, A. Teel, and C. Prieur, "On using norm estimators for eventtriggered control with dynamic output feedback," in IEEE Conference on Decision and Control, 2015, pp. 5500-5505.

[20] M. Abdelrahim, R. Postoyan, J. Daafouz, and D. Nešić, "Stabilization of nonlinear systems using event-triggered output feedback laws," in 21st International Symposium on Mathematical Theory of Networks and Systems, 2014, pp. CD-ROM.

[21] D. P. Borgers and W. P. M. H. Heemels, "Event-separation properties of event-triggered control systems," IEEE Transactions on Automatic Control, vol. 59, no. 10, pp. 2644-2656, 2014.

[22] H. Yu and P. J. Antsaklis, "Event-triggered output feedback control for networked control systems using passivity: Achieving $L_{2}$ stability in the presence of communication delays and signal quantization," Automatica, vol. 49, no. 1, pp. 30-38, 2013.

[23] X. Su, F. Xia, J. Liu, and L. Wu, "Event-triggered fuzzy control of nonlinear systems with its application to inverted pendulum systems ," Automatica, vol. 94, pp. 236-248, 2018.

[24] D. Du, B. Qi, M. Fei, and C. Peng, "Multiple event-triggered $H_{2} / H_{\infty}$ filtering for hybrid wiredcwireless networked systems with random network-induced delays," Information Sciences, vol. 325, no. C, pp. 393 408, 2015.

[25] A. Rahnama, M. Xia, and P. J. Antsaklis, "Passivity-based design for event-triggered networked control systems," IEEE Transactions on Automatic Control, vol. PP, no. 99, pp. 1-1, 2017.

[26] X. Zhong and H. He, "An event-triggered ADP control approach for continuous-time system with unknown internal states," IEEE Trans Cybern, vol. PP, no. 99, pp. 1-12, 2017.

[27] V. S. Dolk, D. P. Borgers, and W. P. M. H. Heemels, "Output-based and decentralized dynamic event-triggered control with guaranteed $\mathcal{L}_{p^{-}}$ gain performance and zeno-freeness," IEEE Transactions on Automatic Control, vol. 62, no. 1, pp. 34-49, 2017.

[28] J. H. Ahrens, X. Tan, and H. K. Khalil, "Multirate sampled-data output feedback control with application to smart material actuated systems," IEEE Transactions on Automatic Control, vol. 54, no. 11, pp. 25182529, 2009.
[29] L. Yao and H. K. Wen, "Design of observer based adaptive PID controller for nonlinear systems," International Journal of Innovative Computing Information and Control Ijicic, vol. 9, no. 2, pp. 667-677, 2013.

[30] H. Bo and G. Wang, "General observer-based controller design for singular markovian jump systems," International Journal of Innovative Computing Information and Control Ijicic, vol. 10, no. 5, pp. 1897-1913, 2014.

[31] M. B. G. Cloosterman, N. V. D. Wouw, W. P. M. H. Heemels, and H. Nijmeijer, "Stability of networked control systems with uncertain time-varying delays," IEEE Transactions on Automatic Control, vol. 54, no. 7, pp. 1575-1580, 2009.

[32] H. P. Wang, Y. Tian, and N. Christov, "Piecewise-continuous observers for linear systems with sampled and delayed output," International Journal of Systems Science, vol. 47, no. 8, 2016.

[33] T. Ahmed-Ali, V. Van Assche, J. F. Massieu, and P. Dorléans, "Continuous-discrete observer for state affine systems with sampled and delayed measurements," IEEE Transactions on Automatic Control, vol. 58, no. 4, pp. 1085-1091, 2013.

[34] M. Nadri and H. Hammouri, "Design of a continuous-discrete observer for state affine systems," Applied Mathematics Letters, vol. 16, no. 6, pp. 967-974, 2003.

[35] F. Celle, J. P. Gauthier, D. Kazakos, and G. Sallet, "Synthesis of nonlinear observers: A harmonic-analysis approach," Mathematical Systems Theory, vol. 22, no. 1, pp. 291-322, 1989.

[36] W. P. M. H. Heemels and M. C. F. Donkers, "Model-based periodic event-triggered control for linear systems," Automatica, vol. 49, no. 3, pp. 698-711, 2013.

[37] A. Girard, "Dynamic triggering mechanisms for event-triggered control," IEEE Transactions on Automatic Control, vol. 60, no. 7, pp. 1992-1997, 2015.

[38] Y. Wang, W. X. Zheng, and H. Zhang, "Dynamic event-based control of nonlinear stochastic systems," IEEE Transactions on Automatic Control, vol. PP, no. 99, pp. 1-1, 2017.

[39] T. Ahmed-Ali, R. Postoyan, and F. Lamnabhi-Lagarrigue, "Continuousdiscrete adaptive observers for state affine systems," Automatica, vol. 45, no. 12, pp. 2986-2990, 2009. 